\title{
Interaction of Colocalized Neuropeptides: Functional Significance in the Circadian Timing System
}

\author{
H. Elliott Albers, ${ }^{1}$ Shyh-Yuh Liou, ${ }^{1, a}$ Edward G. Stopa, ${ }^{2}$ and R. Thomas Zoeller ${ }^{3}$ \\ 'Laboratory of Neuroendocrinology and Behavior, Departments of Biology and Psychology, Georgia State University, \\ Atlanta, Georgia 30303, 'Department of Pathology, State University of New York Health Science Center, Syracuse, New \\ York 13210, and ${ }^{3}$ Department of Anatomy, University of Missouri Medical School, Columbia, Missouri 65212
}

\begin{abstract}
The suprachiasmatic nucleus ( $\mathrm{SCN}$ ), which appears to act as a circadian clock, contains a subpopulation of local circuit neurons in which vasoactive intestinal peptide (VIP), peptide histidine isoleucine (PHI), and gastrin releasing peptide (GRP) are colocalized. To determine whether VIP, PHI, and GRP interact within the SCN to produce a signal important for circadian control, the behavioral and cellular effects of coadministration of these neuropeptides were investigated. Coadministration of VIP, PHI, and GRP within the SCN mimicked the phase-delaying effects of light on circadian control following in vivo microinjection and activated SCN single units recorded in vitro. These behavioral and cellular effects of coadministration of VIP, PHI, and GRP were significantly greater than administration of VIP, PHI, or GRP alone or coadministration of any 2 of these peptides. These data illustrate a new mechanism whereby multiple, colocalized neuropeptides interact in a functionally significant manner, and indicate that the interaction of VIP, PHI, and GRP may be involved in the regulation of circadian rhythms by the SCN.
\end{abstract}

The colocalization of multiple neurotransmitters within the same neuron has been extensively documented in various regions of the CNS and PNS (Hokfelt et al., 1986). Despite the abundance of anatomical data demonstrating the coexistence of neurotransmitters, comparatively little is known about the functional consequences of neurotransmitter corelease. Studies in the PNS, however, have shown that coreleased neurotransmitters can interact to produce a signal different from that achieved by the release of either neurotransmitter alone. For example, vasoactive intestinal peptide (VIP) coreleased with ACh from parasympathetic neurons innervating the cat salivary gland potentiates the secretory activity of $\mathrm{ACh}$, though VIP causes no secretion alone (Lundberg and Hokfelt, 1983). It seems likely that coreleased neurotransmitters will interact in a variety of functionally significant ways, given the different classes of chemical signals that have been colocalized and the widespread distribution of neurons containing more than 1 neurotransmitter.

\footnotetext{
Received July 17, 1990; revised Oct. 30, 1990; accepted Nov. 5, 1990.

This work was supported by Grant N00014-89-J-1640 from the Office of Naval Research to HEA. We thank Earl Anderson and Jay Peller for their technical assistance with these experiments and Drs. Tim Bartness and Barry Ruback for their advice on the manuscript.

Correspondence should be addressed to H. Elliott Albers, Ph.D., at the above address.

a Present address: Upjohn Pharmaceuticals Limited, Tsukuba Research Laboratories, 23 Wadai, Tsukuba-shi, Ibaraki, 300-42, Japan.

Copyright (C) 1991 Society for Neuroscience $0270-6474 / 91 / 110846-06 \$ 03.00 / 0$
}

In the hypothalamus, several variations have been observed in the molecular mechanisms producing colocalized peptides (Sofroniew et al., 1984). In some cases, the colocalized peptides come from the same precursor, while in other cases, the colocalized peptides are derived from different precursors. One population of hypothalamic neurons in which both of these mechanisms appear to be active is in the suprachiasmatic nucleus ( $\mathrm{SCN}$ ). A large subpopulation of local circuit $\mathrm{SCN}$ neurons contains 2 peptides derived from the same precursor, VIP and peptide histidine isoleucine (PHI; Nishizawa et al., 1985) and has been more recently found to contain a third peptide derived from a different precursor, gastrin releasing peptide (GRP; Okamura et al., 1986). The existence of GRP in VIP/PHI-immunoreactive neurons is also consistent with the distributions of GRP and VIP/PHI immunoreactivity and mRNA previously reported within the SCN (Card et al., 1981, 1988; van den Pol and Tsujimoto, 1985; Stopa et al., 1988; Zoeller et al., 1989).

A large body of evidence supports the view that the SCN acts as a circadian clock that both generates rhythms in behavior and physiology and synchronizes those rhythms with the environmental light-dark (LD) cycle (Meijer and Rietveld, 1989). The SCN can be divided into several anatomically distinct subpopulations of intrinsic neurons (van den Pol, 1980; Moore and Card, 1985), but it is not known how these neuronal subpopulations contribute to the generation and synchronization of circadian rhythms with the LD cycle. One hypothesis is that SCN neurons containing VIP/PHI are involved in mediating the effects of environmental lighting important in the synchronization of circadian rhythms with the LD cycle. This hypothesis is supported by the findings that environmental lighting selectively alters VIP and PHI immunoreactivity within the SCN (Albers et al., 1987), and that the cellular levels of VIP/PHI mRNA vary over the day-night cycle (Stopa et al., 1988; Gozes et al., 1989; Albers et al., 1990). The majority of VIP/PHIimmunoreactive neurons are contained within the ventrolateral $\mathrm{SCN}$, which is also the site of termination of most SCN afferent pathways, including 2 well-defined photic projections, the retinohypothalamic tract (RHT) and the geniculohypothalamic tract (GHT) (Moore and Card, 1985). The report that VIP, PHI, and GRP are colocalized in local circuit neurons in the afferent terminal fields of the SCN suggests that synchronization of circadian rhythms with the LD cycle may depend on the corelease of these 3 peptides. If so, the combined effect of VIP, PHI, and GRP on circadian rhythms should be different from the effects of each peptide given either alone or in combination with 1 of the other 2 peptides. The purpose of the present study was to verify the colocalization of VIP/PHI and GRP mRNA in SCN 
neurons using in situ hybridization, and to investigate the functional consequences of VIP, PHI, and GRP colocalization in the control of circadian rhythms by SCN neurons at the behavioral and cellular levels.

\section{Materials and Methods}

In situ hybridization. Rats housed under an LD 14:10 cycle for at least 2 weeks were killed by decapitation. Coronal sections $(12 \mu \mathrm{m})$ through the male rat SCN were made on a cryostat; each section was thaw mounted onto a separate gelatin-coated slide. The steps used for in situ hybridization have been reported previously (Zoeller et al., 1989). In brief, sections were warmed to room temperature, immersed in $4 \%$ formaldehyde/phosphate-buffered saline (PBS) for $5 \mathrm{~min}$, rinsed in PBS, and soaked for $10 \mathrm{~min}$ in $0.25 \%$ acetic anhydride in $0.1 \mathrm{M}$ triethanolamine hydrochloride $/ 0.9 \% \mathrm{NaCl}(\mathrm{pH}, 8.0)$. After rinsing in $2 \times \mathrm{S} \mathrm{SC}$ ( $300 \mathrm{~mm} \mathrm{NaCl} / 30 \mathrm{~mm}$ sodium citrate), sections were dehydrated through a graded series of ethanol, delipidated in chloroform, rehydrated to $95 \%$ ethanol, and air dried. The hybridization buffer contained $50 \%$ formamide, $4 \times$ SSC, transfer RNA $(250 \mu \mathrm{g} / \mathrm{ml})$ sheared single-stranded salmon sperm DNA $(100 \mu \mathrm{g} / \mathrm{ml}), 1 \times$ Denhardt's solution $(0.02 \%$ each of BSA, Ficoll, and polyvinylpyrrolidone), $10 \%(\mathrm{w} / \mathrm{v})$ dextran sulfate (MW, 500,000), $100 \mathrm{~mm}$ dithiothreitol (DDT), and $0.75 \times 10^{6} \mathrm{cpm}$ probe. Fifty microliters of hybridization buffer was applied to each slide, which was then covered with a parafilm coverslip and incubated at $37^{\circ} \mathrm{C}$ for $20 \mathrm{hr}$ in humid chambers. Following hybridization, the coverslips were floated off in $1 \times \mathrm{SSC}$ and washed 2 times in $1 \times \mathrm{SSC}$, then washed 4 times for $15 \mathrm{~min}$ each in $2 \times \mathrm{SSC} / 50 \%$ formamide at $40^{\circ} \mathrm{C}$. After 2 30 -min washes in $1 \times$ SSC at room temperature, the slides were dipped in distilled water, equilibrated in $70 \%$ ethanol, and air dried.

The 48-base oligonucleotides were $3^{\prime}$ end labeled using terminal deoxynucleotidyl transferase. The base sequence for VIP/PHI (ATGGAGTTCAAGTATTTCTTCACAGCCATTTGCTTTCTAAGGCGGGT) corresponds to base numbers $403-449$ (see Nishizawa et al., 1985). The base sequence for GRP (CCCTTCAGCTGGCAGTTCCTTCCTTCCCCTTGAGAACCTGGAGCAGAG) corresponds to the sequence used previously to map the distribution of GRP mRNA in the rat brain (Zoeller et al., 1989).

Microinjection experiments. Hamsters were used for behavioral studies examining the interaction of VIP, PHI, and GRP because of the precise nature of their wheel-running rhythm and because the anatomy of the rat and hamster are very similar (Card and Moore, 1984). Hamsters were deeply anesthetized with sodium pentobarbital and stereotaxically implanted with 26-gauge guide cannulas aimed at the suprachiasmatic region. The stereotaxic coordinates were $1.6 \mathrm{~mm}$ anterior and $1.7 \mathrm{~mm}$ lateral to bregma, and $-7.7 \mathrm{~mm}$ below dura. The incisor bar was set at 0 , and the stereotaxic arm was set $10^{\circ}$ from perpendicular. Following surgery, each hamster was placed in a cage containing an activity wheel in a room maintained under continuous illumination (700-1000 lux). After a stable free-running activity rhythm was established, peptides were microinjected into the SCN region at 7-10-d intervals. Peptides were dissolved in $0.9 \% \mathrm{NaCl}$ (saline) and injected with a 1- $\mu 1$ Hamilton syringe connected to a 33-gauge needle by polyethylene tubing. Peptides were purchased from Bachem, Inc., Torrance, CA. A cocktail containing approximately equimolar concentrations of VIP, PHI, and GRP was made by dissolving approximately 50 pmol VIP, $50 \mathrm{pmol}$ PHI, and $50 \mathrm{pmol}$ GRP into $400 \mathrm{nl}$ saline. In all protocols, a total of $150 \mathrm{pmol}$ peptide was injected in $400 \mathrm{nl}$ saline. Following the experiment, hamsters were deeply anesthetized with sodium pentobarbital and microinjected with $400 \mathrm{nl}$ of dye. Following intracardial perfusion with Perfix (Fisher Scientific), the brain was removed and examined to verify the site of injection. Phase shifts in the activity rhythm were calculated by linear regression using the method of Daan and Pittendrigh (1976).

Hypothalamic slice experiments. Extracellular single-unit recordings were made from SCN slices harvested from hamsters housed in an LD 14:10 cycle using procedures reported previously (Liou and Albers, 1989). In brief, coronal brain slices $400-450 \mu \mathrm{m}$ in thickness were cut on a vibratome and placed in incubation medium oxygenated with $95 \%$ $\mathrm{O}_{2}$ and $5 \% \mathrm{CO}_{2}$ at $35^{\circ} \mathrm{C}$ for $1 \mathrm{hr}$. Slices were then transferred to the recording chamber, where extracellular recordings were made using glass micropipettes with DC resistances of $25-40 \mathrm{M} \Omega$ filled with $0.5 \mathrm{M}$ sodium acetate containing $2 \%$ fast green. Repeated experiments were conducted on the same unit only if the firing rate returned to baseline after the washout period. The responses to application of peptides were assessed in the integrated records obtained from a chart recorder by calculating the change in firing rate from the resting level (mean firing rate during the $5 \mathrm{~min}$ prior to drug application) to the peak level (mean firing rate during $1 \mathrm{~min}$ ). Peptide-containing medium reached the recording chamber 2-3 min after the switch from incubation medium. At the end of the recording session, a current of $5 \mu \mathrm{A}$ was passed through the electrode for 3-5 min to deposit a blue spot. All recording sites used in the present study were in the ventrolateral SCN, where the VIP-, PHI-, and GRPimmunoreactive neurons are primarily located.

\section{Results}

The colocalization of VIP/PHI and GRP in SCN neurons was examined in rats by comparison of VIP/PHI and GRP mRNA within neurons of the ventrolateral SCN using in situ hybridization. As expected, the autoradiographic signal produced by probes complementary to rat VIP/PHI and GRP mRNAs was restricted to the ventrolateral aspect of the SCN. Analysis of adjacent sections labeled separately for VIP/PHI mRNA or GRP mRNA indicated that GRP mRNA and VIP/PHI mRNA could be found in the same SCN neuron (Fig. 1). It was not possible to estimate the proportion of VIP/PHI neurons containing GRP because the section thickness $(12 \mu \mathrm{m})$ was greater than the average diameter of nuclei in SCN neurons (i.e., $6.51 \pm 0.8 \mu \mathrm{m}$ ); thus, not all nuclei were contained within both sections. However, because the low abundance of GRP mRNA requires several weeks of exposure to detect the signal, thinner sections would have compromised the ability to detect GRP mRNA in single cells. The long exposure time necessary for the detection of GRP mRNA suggests that the mRNA encoding GRP is expressed less abundantly than VIP/PHI mRNA.

Because these and other data (Okamura et al., 1986) indicate that VIP, PHI, and GRP are colocalized within a subpopulation of SCN neurons, the circadian effects of coadministration of these peptides on the timing of circadian rhythms were examined in hamsters. As can be seen in Figure 1, microinjection of VIP/PHI/GRP produced large phase delays in the timing of the circadian activity rhythm when administered around the time of the onset of the daily active phase, but not at other times within the circadian cycle. The phase shifts produced by microinjection of VIP/PHI/GRP throughout the circadian cyclc $(N=39)$ are summarized in Figure 2 . The magnitude of the phase shifts produced by VIP/PHI/GRP differed significantly $(p<0.05)$ depending on time of administration within the circadian cycle. No corresponding differences $(p>0.05)$ were observed in the effects of VIP/PHI/GRP on the free-running circadian period over the circadian cycle. Comparison of the effects of VIP/PHI/GRP on circadian timing with the well-known phase-shifting effects of brief light pulses (Daan and Pittendrigh, 1976) revealed that VIP/PHI/GRP mimicked the phase-delaying, but not the phase-advancing, effects of light.

To investigate whether the phase delays produced by VIP/ PHI/GRP were the result of their action within the SCN region, or whether these peptides may have acted in CNS regions other than the SCN after entering the ventricular system, the effects of VIP/PHI/GRP microinjection into the SCN region were compared with microinjections given directly into the lateral ventricle. VIP/PHI/GRP administered into the lateral ventricle during the 3-hr interval following the onset of activity (i.e., circadian time $12-15)$ produced a mean $( \pm$ SEM) phase shift of $0.04 \pm 0.13 \mathrm{hr}(N=8)$, which differed significantly $(p<0.01)$ from the $1.53 \pm 0.35(N=11)$ phase delay produced by VIP/ $\mathrm{PHI} / \mathrm{GRP}$ microinjected into the SCN region. These data there- 

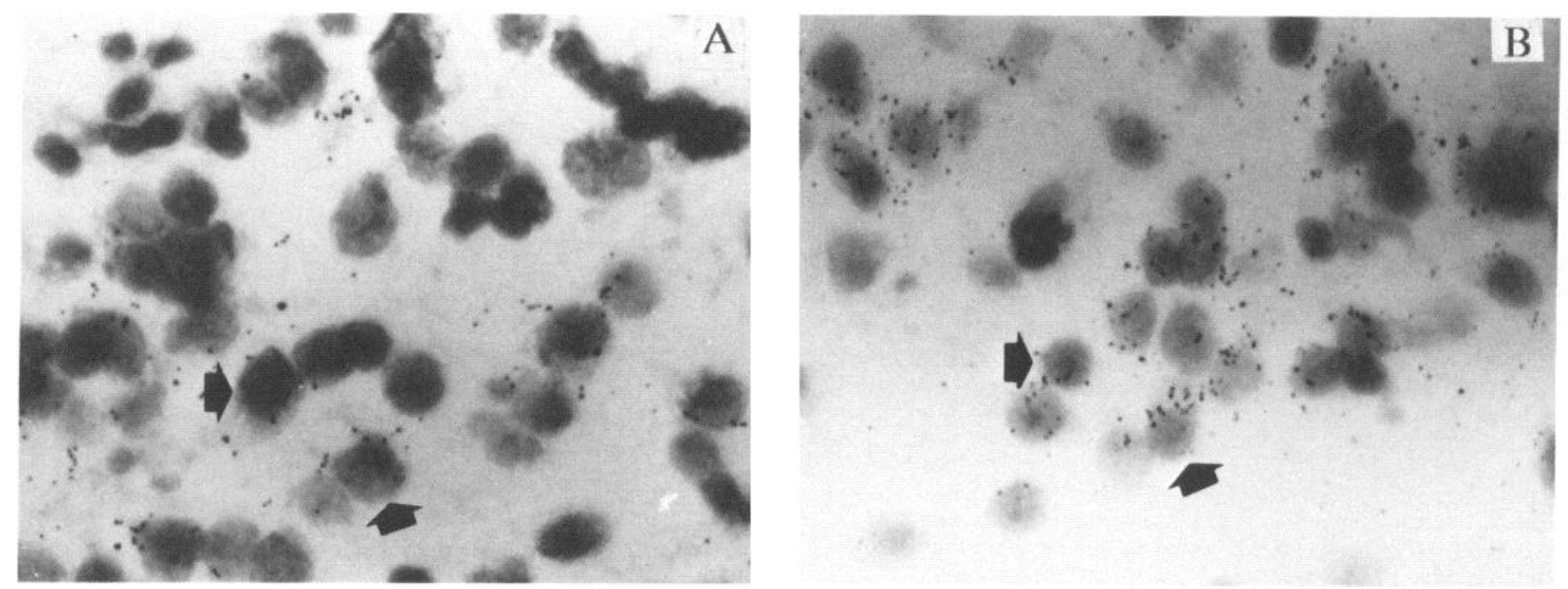

Figure 1. Colocalization of mRNAs encoding VIP/PHI and GRP in neurons of the rat SCN: bright-field micrograph of SCN neurons contained within adjacent sections and visualized with ${ }^{35} \mathrm{~S}$-labeled oligonucleotides complementary to mRNAs encoding VIP/PHI $(A)$ or GRP $(B)$. Individual nuclei are stained with toluidine blue. The presence of a specific mRNA is indicated by silver grains in the photographic emulsion over a nucleus. Nuclei labeled by both probes in adjacent sections (arrows) indicate neurons that contain both VIP/PHI and GRP mRNA. Magnification: $800 \times$.

fore support the view that the phase-shifting effects of VIP/PHI/ GRP result from their influence on SCN neurons.

Although VIP/PHI/GRP coexist within a subpopulation of $\mathrm{SCN}$ neurons, it is not known whether an interaction between all 3 peptides is required to mimic the phase-delaying effects of light, or if 1 or 2 of these peptides is sufficient to phase delay circadian rhythms. To investigate the potential significance of the interaction among VIP, PHI, and GRP within the SCN, the phase-shifting effects of administration of VIP, PHI, and GRP and the coadministration of VIP/PHI, VIP/GRP, and PHI/GRP were compared with the coadministration of VIP/PHI/GRP. In each injection, the total amount of peptide $(150 \mathrm{pmol})$ given was identical (e.g., the VIP injections contained $150 \mathrm{pmol}$ of VIP, and the VIP/PHI/GRP injections contained 50 pmol VIP, 50 pmol PHI, and 50 pmol GRP). A statistically significant ( $p$ $<0.05$ ) difference was observed in the magnitude of the phase delays produced by the various combinations of the 3 peptides (Fig. 3), but no corresponding differences $(p>0.05)$ were observed in the free-running circadian period. Administration of VIP, PHI, or GRP alone phase delayed the activity rhythm by 30 min or less. Although coadministration of 2 of the 3 peptides produced slightly larger phase delays than when given alone, the phase delays produced by coadministration of 2 peptides remained at least $50 \%$ less than those produced by coadministration of VIP/PHI/GRP.

The next series of experiments used the hypothalamic slice preparation and examined the cellular effects of VIP, PHI, and GRP and their coadministration on SCN neurons. The spontaneous firing of neurons in the ventrolateral $\mathrm{SCN}$ was recorded extracellularly during an 8-hr interval beginning at the phase of the circadian cycle corresponding to $4 \mathrm{hr}$ before the time that locomotor activity normally begins (i.e., circadian time 8-16). Coadministration of VIP/PHI/GRP into the perfusate increased the firing rate by an average of $3.85 \pm 0.35$ impulses/sec in $65 \%$ of the 23 neurons examined. In contrast, coadministration of any 2 of the peptides, or VIP, PHI, or GRP alone, in the same molar concentration (i.e., $10^{-7}$ ), produced significantly less excitation than did coadministration of VIP/PHI/GRP (Figs. 4,
5). The excitation of $\mathrm{SCN}$ neurons produced by peptide coadministration was dose dependent (Fig. 6).

\section{Discussion}

The coadministration of VIP/PHI/GRP is necessary for the full expression of the cellular and behavioral effects of these peptides within the SCN. VIP, PHI, or GRP, or any combination of 2 of these peptides, did not produce phase delays or an increase in single-unit discharge of the magnitude produced by combined administration of all 3 peptides. Furthermore, there was no evidence that any one of these peptides represents the primary signal of the VIP/PHI/GRP SCN neurons, because no differences were observed in the effects of these peptides when given alone or in combination with one of the other peptides. These observations suggest that VIP, PHI, and GRP interact within the SCN by a mechanism that is different from that previously reported for other colocalized messengers, where one neurotransmitter usually constitutes the primary signal, while the other messenger modifies that signal but has little or no activity alone (Hokfelt et al., 1986).

The present findings provide strong evidence for the localization of specific circadian functions to a subpopulation of intrinsic SCN neurons. The ability of VIP/PHI/GRP to mimic the phase-delaying effects of light on circadian rhythms within the SCN supports the hypothesis that the neurons that contain these neuropeptides are involved in the synchronization of circadian rhythms by the LD cycle. VIP/PHI/GRP-containing neurons may also integrate more than just photic input to the $\mathrm{SCN}$ because photic as well as nonphotic projections terminate directly on VIP-immunoreactive SCN neurons (Kiss et al., 1984; Bosler and Beaudet, 1985; Ibata et al., 1987).

One hypothesis for how VIP/PHI/GRP SCN neurons integrate afferent information is that the availability of the 3 peptides within SCN neurons is differentially regulated by afferent input. If so, the ratio of the peptides available for release would be altered depending on the specific type of afferent information received. Other data indicate that afferent neuronal activity can influence the expression of specific neurosecretory products (Ei- 
TIME OF DAY (HRS)
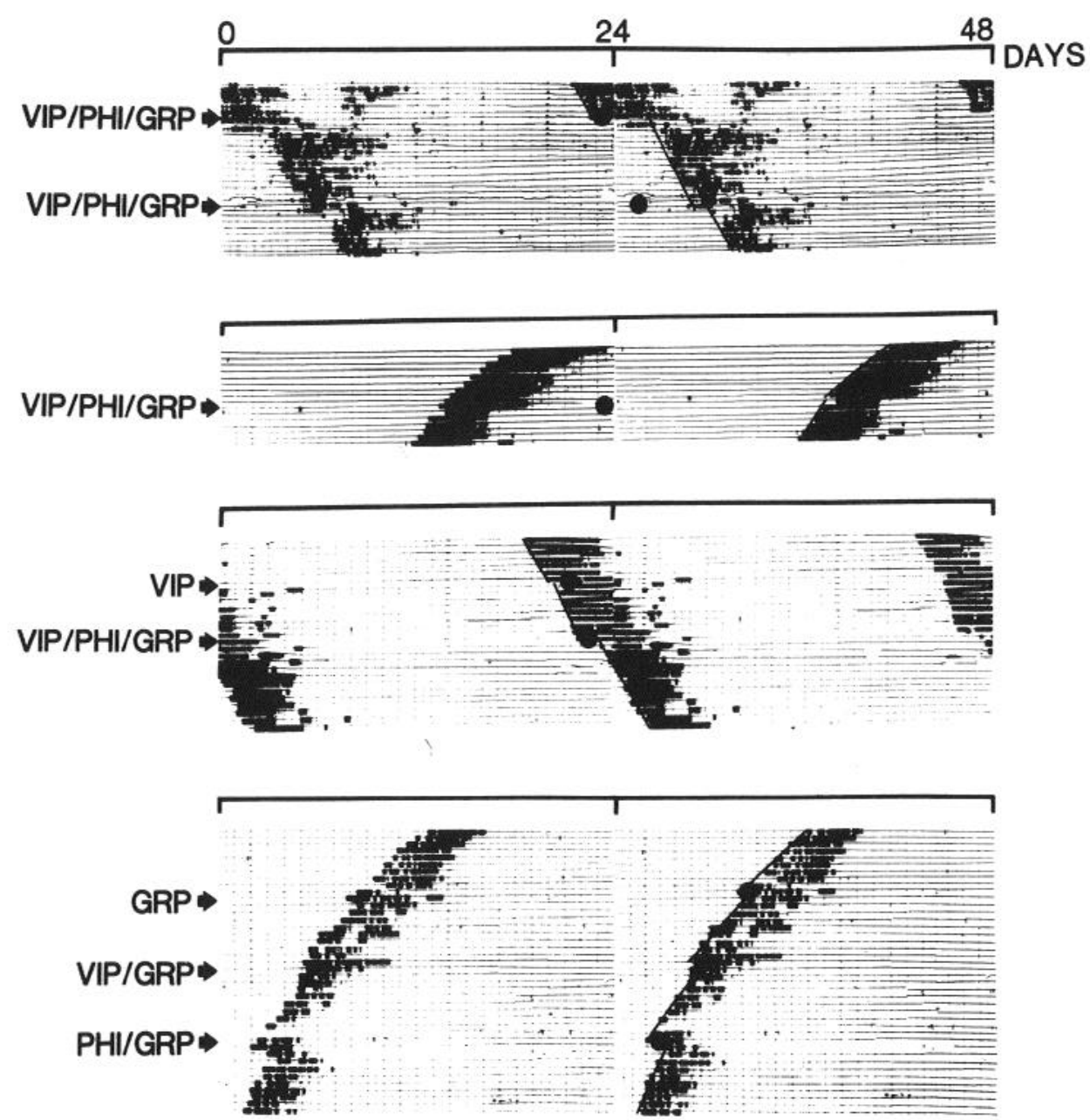

Figure 2. Effect of various combinations of VIP, PHI, and GRP on the phase of free-running activity rhythms following their microinjection into the suprachiasmatic region of the hamster. Coadministration of VIP $(50 \mathrm{pmol})$, PHI (50 pmol), and GRP (50 pmol) produced large phase delays when given around the time of activity onset, but had little effect at other times within the circadian cycle. Microinjection of VIP (150 pmol) or GRP (150 pmol), or coadministration of VIP $(75 \mathrm{pmol})$ and GRP (75 pmol) or PHI (75 pmol) and GRP (75 pmol), produced only small delays in circadian phase.

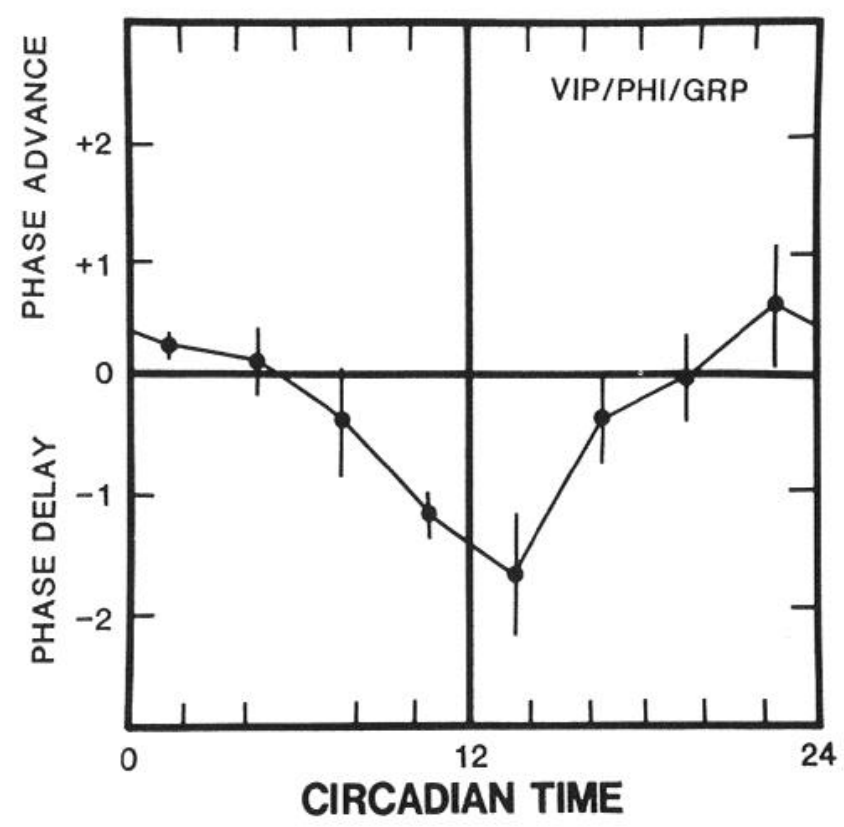

Figure 3. Summary of the effects of microinjections of VIP/PHI/GRP $(N=39)$ into the suprachiasmatic region plotted as a phase-response curve. Solid circles represent mean phase shifts $( \pm$ SEM), in hours, at 3 -hr intervals throughout the circadian cycle (circadian time 12 refers den et al., 1984; Friedman et al., 1988). Furthermore, cellular levels of VIP/PHI mRNA are not constant within the SCN but vary such that greater concentrations occur at night than during the day (Stopa et al., 1988; Gozes et al., 1989; Albers et al., 1990). Interestingly, cellular levels of GRP mRNA within the $\mathrm{SCN}$ also change over the day, but in a pattern distinctly different from that of VIP/PHI mRNA (Zoeller et al., 1990). Collectively, these data indicate that availability of these peptides may be differentially regulated, and, as a result, the ratio of VIP/PHI to GRP released within the SCN might change as a function of time of day and/or afferent input. The results of the present study suggest that, if the changes in the ratios of VIP, PHI, and GRP are sufficiently large, the resulting circadian effects (e.g., magnitude of phase delay) produced by the endogenously released neuropeptide cocktail may be correspondingly altered.

It is not known why the coadministration of VIP/PHI/GRP mimics the phase-delaying but not the phase-advancing effects of light pulses. One possibility is that phase advances and phase

to the time of activity onset). VIP/PHI/GRP produced phase-delay shifts around the time of activity onset, but had no effect at other times within the circadian cycle. VIP/PHI/GRP microinjection mimics the phase-delaying but not the phase-advancing effects of brief light pulses (Daan and Pittendrigh, 1976). 


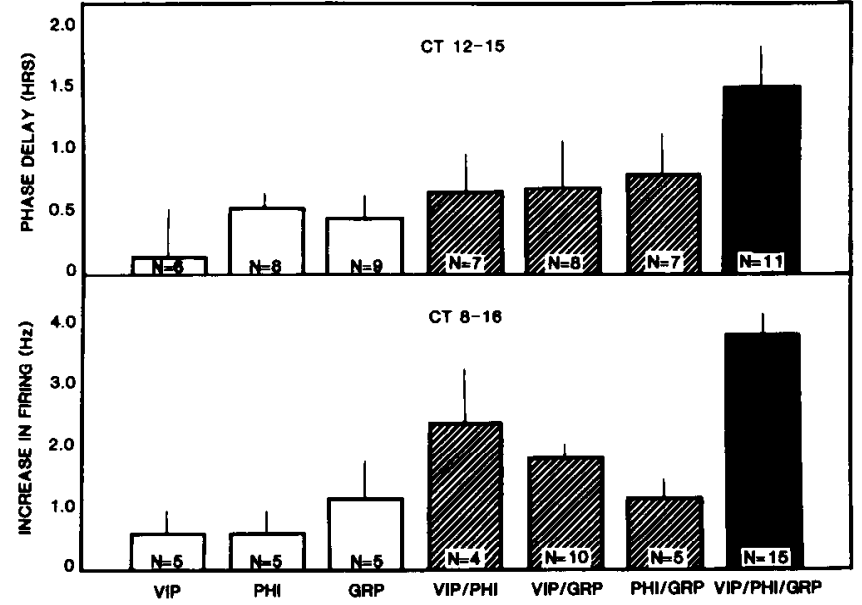

Figure 4. Summary of the behavioral and cellular effects of administration of VIP, PHI, and GRP alone, coadministration of 2 of these peptides (i.c., VIP/PHI, VIP/GRP, and PHI/GRP), and coadministration of all 3 peptides (VIP/PHI/GRP). TOP, Phase shifts produced in hamster wheel-running rhythms following peptide microinjection into the suprachiasmatic region given in the $3 \mathrm{hr}$ following activity onset (i.e., circadian time 12-15). One-way ANOVA revealed a significant difference $(p<0.05)$ in the magnitude of phase delays between the groups. Post hoc comparisons subsequent to the ANOVA revealed statistically significant $(p<0.05)$ differences between the VIP/PHI/GRP group and the VIP, PHI, GRP, VIP/PHI, and VIP/GRP groups. A priori comparisons indicated a statistically significant $(p<0.05)$ difference between the VIP/PHI/GRP group and the VIP + PHI + GRP groups and between the VIP/PHI/GRP group and the VIP/PHI + VIP/GRP + PHI/GRP groups. All statistics were calculated using SPSSx. Bottom, Increase in spontaneous discharge of SCN single units following administration of VIP, PHI, and GRP and coadministration of all possible combinations of these peptides in a final concentration of $10^{-7} \mathrm{M}$. All single-unit recordings were made during an 8-hr interval (circadian

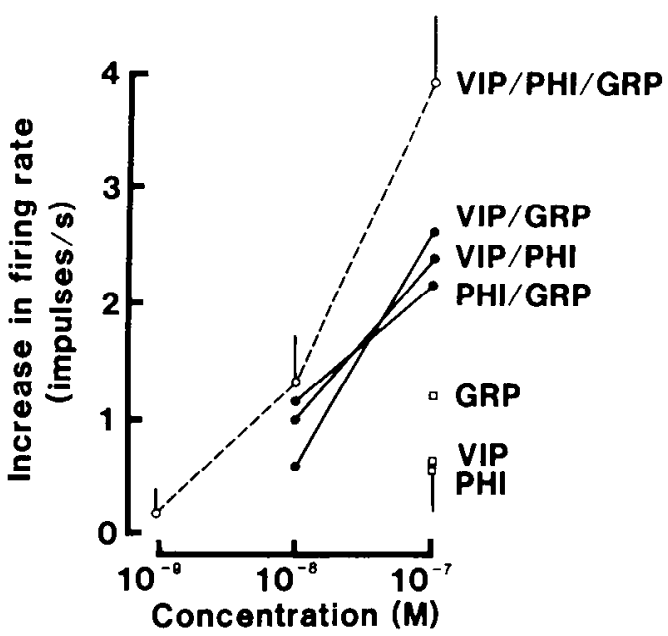

Figure 6. Dose-response of SCN single units to coadministration of VIP/PHI/GRP $(N=5), \mathrm{VIP} / \mathrm{PHI}(N=4), \mathrm{VIP} / \mathrm{GRP}(N=3), \mathrm{PHI} / \mathrm{GRP}$ $(N=3)$, VIP $(N=5)$, PHI $(N=5)$, and $\operatorname{GKP}(N=5)$. Error bars represent SEM.

time 8-16) of the circadian cycle beginning $4 \mathrm{hr}$ before the phase corresponding to activity onset. A significant $(p<0.001)$ difference in the increase in firing was observed between the groups. Post hoc subsequent tests revealed a significant $(p<0.05)$ difference between the VIP/PHI/ GRP group and each of the other 6 groups. In addition, a priori subsequent tests indicated a statistically significant difference $(p<0.01)$ between the VIP/PIII/GRP group and the VIP + PHI + GRP groups and between the VIP/PHI/GRP group and the VIP/PHI / VIP/GRP + PHI/GRP groups. Error bars represent SEM.
A

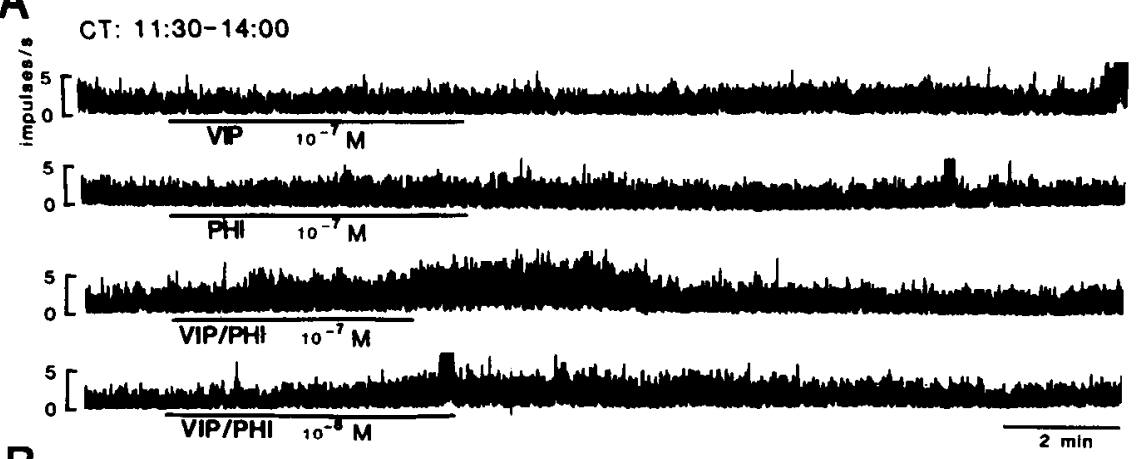

B
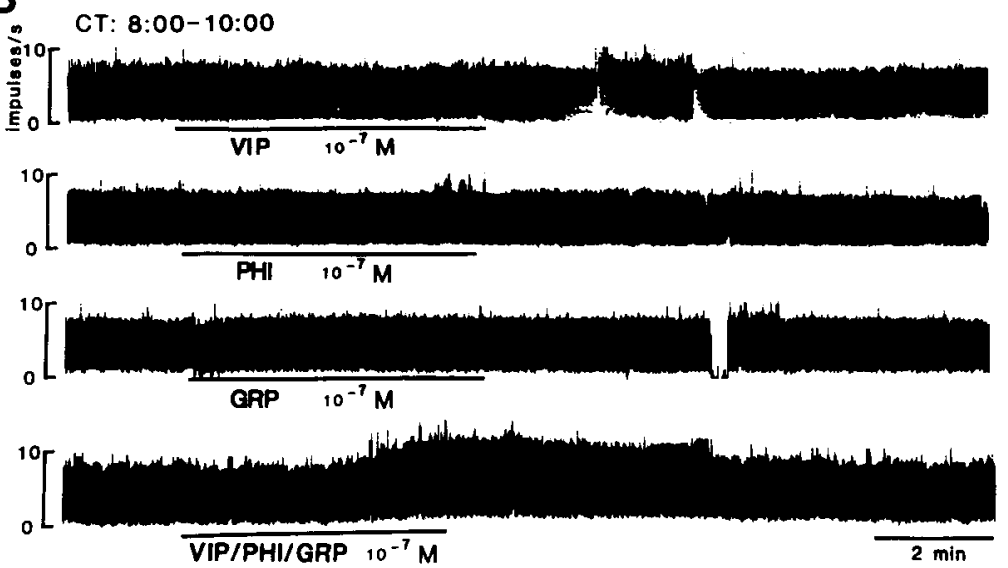

Figure 5. Integrated firing-rate records from $2 \mathrm{SCN}$ neurons recorded extracellularly in the hypothalamic slice preparation. $A$, Response of an SCN neuron to VIP, PHI, and coadministration of VIP/PHI. $B$, Response of an SCN neuron to VIP, PHI, GRP, and coadministration of VIP/PHI/GRP. 
delays are mediated by different neurochemical pathways. This hypothesis was originally proposed following the demonstration that systemic injection of the GABA receptor antagonist bicuculline blocked light-pulse-induced phase delays, but not phase advances (Ralph and Menaker, 1986). This view is also consistent with the findings that glutamate and neuropeptide $Y$, which may be contained in the photic projections to the SCN, produce phase advances, but little or no phase delays, when microinjected into the SCN (Albers and Ferris, 1984; Albers et al., 1984; Meijer et al., 1988). An alternative hypothesis is that the ratio of VIP/PHI/GRP released within the SCN is critical in defining the circadian effects of these peptides. According to this view, it might be possible to induce phase-advance shifts by microinjection of the correct ratio of VIP/PHI/GRP cocktail into the SCN.

\section{References}

Albers HE, Ferris CF (1984) Neuropeptide Y: role in light-dark cycle entrainment of hamster circadian thythm. Neurosci Lett 50:163-168.

Albers HE, Ferris CF, Leeman SE, Goldman BD (1984) Avian pancreatic polypeptide phase shifts hamster circadian rhythms when microinjected into the suprachiasmatic region. Science 223:833-835.

Albers HE, Minamitani N, Stopa E, Ferris CF (1987) Light selectivity alters vasoactive intestinal peptide and peptide histidine isoleucine immunoreactivity within the rat suprachiasmatic nucleus. Brain Res 437:189-192.

Albers HE, Stopa EG, Zoeller RT, Kauer JS, King JC, Fink JS, Mobtaker $\mathrm{H}$, Wolfe $\mathrm{H}$ (1990) Day-night variation in prepo vasoactive intestinal peptide/peptide histidine isoleucine mRNA within the rat suprachiasmatic nucleus. Mol Brain Res 7:85-89.

Bosler O, Beaudet A (1985) VIP neurons as prime synaptic targets for serotonin afferents in rat suprachiasmatic nucleus: a combined radioautographic and immunocytochemical study. J Neurocytol 14:749763.

Card JP, Moore RY (1984) The suprachiasmatic nucleus of the golden hamster: immunohistochemical analysis of cell and fiber distribution. Neuroscience 13:415-431.

Card JP, Brecha N, Karten HJ, Moore RY (1981) Immunocytochemical localization of vasoactive intestinal polypeptide-containing cells and processes in the suprachiasmatic nucleus of the rat: light and electron microscopic analysis. J Neurosci 1:1289-1303.

Card JP, Fitzpatrick-McElligott S, Gozes I, Baldino F Jr (1988) Localization of vasopressin, vasoactive intestinal polypeptide-, peptide histidine isoleucine- and somatostatin-mRNA in rat suprachiasmatic nucleus. Cell Tissue Res 252:307-315.

Daan S, Pittendrigh CS (1976) A functional analysis of circadian pacemakers in nocturnal rodents. II. The variability of phase response curves. J Comp Physiol 106:253-266.

Eiden LE, Giraud P, Dave JR, Hotchkiss J, Hans-Urs A (1984) Nicotinic receptor stimulation activates enkephalin release and biosynthesis in adrenal chromaffin cells. Nature 312:661-666.

Friedman WJ, Dreyfus CF, McEwen B, Black IB (1988) Presynaptic transmitters and depolarizing influences regulate development of the substantia nigra in culture. J Neurosci 8:3616-3623.
Gozes I, Shani Y, Liu B, Burbach JPH (1989) Diurnal variation in vasoactive intestinal peptide messenger RNA in the suprachiasmatic nucleus of the rat. Neurosci Res Commun 5:83-86.

Hokfelt T, Fuxe K, Pernow B (1986) Progress in brain research. Amsterdam: Elsevier.

Ibata Y, Takahaski Y, Okamura H, Kawakami F, Terubayashi H, Kubo T, Yanaihara N (1987) Vasoactive intestinal peptide (VIP)-like immunoreactive neurons located in the rat suprachiasmatic nucleus receive a direct retinal projection. Neurosci Lett 97:1-5.

Kiss J, Leranth C, Halasz B (1984) Serotoninergic endings of VIPneurons in the suprachiasmatic nucleus and on ACTH-neurons in the arcuate nucleus of the rat hypothalamus. A combination of high resolution autoradiography and electron microscopic immunocytochemistry. Neurosci Lett 44:119-124.

Liou SY, Albers HE (1989) Single unit response of suprachiasmatic neurons to arginine vasopressin (AVP) is mediated by a $\mathrm{V}_{1}$-like receptor in the hamster. Brain Res 477:336-343.

Lundberg JM, Hokfelt T (1983) Coexistence of peptides and classical neurotransmitters. Trends Neurosci 6:325-333.

Meijer JH, Rietveld WJ (1989) Neurophysiology of the suprachiasmatic circadian pacemaker in rodents. Physiol Rev 69:67l-707.

Meijer JH, van der Zee EA, Dietz M (1988) Glutamate phase shifts circadian activity rhythms in hamsters. Neurosci Lett 86:177-183.

Moore RY, Card JP (1985) Visual pathways and the entrainment of circadian rhythms. Ann NY Acad Sci 453:123-133.

Nishizawa M, Hayakawa Y, Yanaihara N, Okamoto H (1985) Nucleotide sequence divergence and functional constraint in VIP precursor mRNA evolution between human and rat. FEBS Lett 183:5559.

Okamura H, Murakami S, Uda K, Sugano T, Takahashi Y, Yanaihara C, Yanaihara N, Ibata Y (1986) Coexistence of vasoactive intestinal peptide (VIP)-, peptide histidine isoleucine amide (PHI)-, and gastrin releasing peptide (GRP)-like immunoreactivity in neurons of the rat suprachiasmatic nucleus. Biomed Res 7:295--299.

Ralph MR, Menaker M (1986) Effects of diazepam on circadian phase advances and delays. Brain Res 372:405-408.

Sofroniew MV, Eckenstein F, Schrell V, Cuello AC (1984) Evidence for colocalization of neuroactive substances in hypothalamic neurons. In: Coexistence of neuroactive substances in neurons (Chan-Palay V, Palay SI, eds), pp 73-90. New York: Wiley.

Stopa EG, Minamitani N, Jonassen JA, King JC, Wolfe H, Mobtaker $\mathbf{H}$, Albers HE (1988) Localization of vasoactive intestinal peptide and peptide histidine isoleucine immunoreactivity and mRNA within the rat suprachiasmatic nucleus. Mol Brain Res 4:319-325.

van den Pol AA, Tsujimoto KL (1985) Neurotransmitters of the hypothalamic suprachiasmatic nucleus: immunocytochemical analysis of 25 neuronal antigens. Neuroscience 15:1049-1086.

van den Pol AN (1980) The hypothalamic suprachiasmatic nucleus of rat: intrinsic anatomy. J Comp Neurol 191:661-702.

Zoeller RT, Lebacq-Verheyden AM, Battey JF (1989) Distribution of two distinct messenger ribonucleic acids encoding gastrin-releasing peptide in rat brain. Peptides 10:415-422.

Zoeller RT, Licklider NF, Anderson ER, Albers HE (1990) Cellular levels of messenger RNAs encoding VIP/PHI and gastrin releasing peptide (GRP, bombesin) exhibit different $24 \mathrm{hr}$ rhythms in the rat SCN. Program Endocr Soc 72:336. 